CARDIOVASCULAR MEDICINE

\title{
Depression after myocardial infarction is a risk factor for declining health related quality of life and increased disability and cardiac complaints at 12 months
}

\author{
P de Jonge, T A Spijkerman, R H S van den Brink, J Ormel

See end of article for authors' affiliations

Correspondence to: Dr Peter de Jonge, Department of Psychiatry, University of Groningen, PO Box 30.001, 9700 RB Groningen, Netherlands; p.de.jonge@med.rug.nl

Accepted 4 May 2005 Published Online First 12 May 2005
Objective: To study the prospective effects of post-myocardial infarction (MI) depressive disorder on health status, including self reported cardiac symptoms, disability, and health related quality of life, at 12 months after $\mathrm{MI}$ while controlling for cardiac condition and health status at three months after $\mathrm{MI}$.

Methods: Longitudinal study of a cohort of 468 adults with MI recruited from four hospitals in the north of the Netherlands between September 1997 and September 2000. Assessment of depressive disorder in the year after Ml according to International classification of disease, 10th revision criteria. Assessment of health status at three and 12 months by the RAND 36 item health survey, the health complaints scale, self rated cardiac complaints, and disability.

Results: Patients with post-MI depressive disorder were more likely than those without to have poor quality of life, more health complaints, more cardiac complaints, and more disability at 12 months' follow up. In multivariate analyses adjusted for cardiac condition, health status at three months, age, sex, and pre-Ml depression, the prospective association of post-MI depression with poor health status remained for most of the indicators. Severity of the post-MI depression further contributed to aspects of poor health status.

Conclusions: In a prospective study design, post-MI depression had strong effects on poor health status exceeding the effects of cardiac condition and its short term consequences. Efforts to improve health status after Ml should therefore include standard assessment and guideline based treatment of post-MI depressive disorder.
A fter the acute treatment phase of myocardial infarction (MI) during hospitalisation, the primary treatment goal becomes improvement of health status, including cardiac related symptoms, health related quality of life (QoL), and functional status. ${ }^{1-5}$ Post-MI depression may complicate this treatment goal. ${ }^{6-8}$ In physically healthy people, depression has an effect on functioning and QoL that equals most of the common chronic illnesses. ${ }^{9}$ In patients with a chronic medical illness depression has an additive effect on functional limitations. ${ }^{10}$ Elsewhere, we have reported that depression is a risk factor for poor adjustment to somatic illness events in the elderly, including MI and congestive heart failure, which was reflected by poorer QoL and functional status in the year after the acute illness episode. ${ }^{11}$

Several authors have reported associations of depression after MI with aspects of QoL and functioning. ${ }^{12-15}$ However, whether the effects of depression are independent of baseline functioning and cardiovascular disease severity and its consequences remains unclear. ${ }^{15}$ A recent cross sectional study ${ }^{1}$ reported that depressive symptoms were consistently associated with several aspects of poor functioning and QoL, whereas left ventricular ejection fraction (LVEF) and ischaemia were not. However, since the study did not assess the prospective effect of depression on QoL after MI, it cannot be concluded that depression is a genuine risk factor; the reverse may also be true, or depression and poor QoL may be caused by a common factor such as cardiac related physical disability. As a second limitation of that and other studies, ${ }^{12-15}$ the assessment of depression was based on self report questionnaires rather than according to formal diagnostic criteria. As a result, the possibility of a self report bias, which may be present both in the self reported QoL and the self reported depressive symptoms, cannot be ruled out and a spurious exposure-outcome association may be generated. ${ }^{16}{ }^{17}$

In the present study, we hypothesised that post-MI depression, defined as a depressive disorder according to formal diagnostic criteria after discharge from the hospital, predicts several aspects of health status-cardiac symptoms, poor health related QoL, and disability-at one year after an MI. We expected these effects to be largely independent of baseline cardiac condition, health status at three months after an MI, sociodemographic variables, and depression that was present already before the MI. In addition, we studied the relative impact of duration and severity of the post-MI depression on health status.

\section{METHODS}

\section{Patients and setting}

The DepreMI study is an observational cohort study of depression, QoL, and cardiovascular outcomes after MI. Patients entered the study after being admitted for an MI between September 1997 and September 2000. Patients were found eligible if they met two of three criteria: chest pain for at least 20 minutes, creatine kinase concentration 100\% higher than normal or creatine kinase MB fraction greater than $10 \%$; or the presence of new pathological Q wave on the

Abbreviations: $\mathrm{BDI}$, Beck depression inventory; $\mathrm{Cl}$, confidence interval; CIDI-Auto, composite international diagnostic interview; $\mathrm{HCS}$, health complaints scale; IQOLA, international quality of life assessment; LVEF, left ventricular ejection fraction; $\mathrm{MI}$, myocardial infarction; $\mathrm{OR}$, odds ratio; QoL, quality of life; SPECT, single photon emission computed tomography 
ECG in at least two leads. Exclusion criteria were life expectancy of less than one year due to non-cardiovascular disease, cognitive dysfunction, not being able to speak or read Dutch, too poor physical condition according to hospital staff, and hospital admission for a reason other than MI except angina pectoris. All patients participating in the study signed an informed consent form. The study protocol was approved by the ethics committee review board at the four participating hospitals in the northern the Netherlands: University Hospital Groningen, Martini Hospital Groningen, St Lucas Hospital Winschoten, and Refaja Hospital Stadskanaal.

\section{Measures \\ Sociodemographic data}

Age and sex were assessed during admission. Level of education, living situation, and smoking were assessed in a face to face patient interview three months after an MI. Three levels of education were distinguished: (1) primary school as the highest level of education (low); (2) vocational training for 12-18 year olds or secondary education (medium); and (3) advanced professional training for $\geqslant 18$ year olds or university level of education (high).

\section{Assessment of cardiac condition}

As indicators for cardiac condition, we rated LVEF, whether the patient had a previous MI, and whether the patient had heart failure. The presence of heart failure was indicated by Killip class at admission (that is, a standardised four point clinical assessment of the degree of heart failure based on pulmonary rales and radiography, divided as class $\geqslant$ II or $<$ II). LVEF was assessed by nuclear method, gated single photon emission computed tomography (SPECT), wall motion score index, magnetic resonance imaging, angiography, or clinical judgment by the treating cardiologist and dichotomised as $\geqslant 40 \%$ or $<40 \%$. For the patients ( 267 of $421(63.4 \%))$ for whom we had LVEF scores based on nuclear method, gated SPECT, wall motion score, magnetic resonance imaging, or angiography, the original continuous LVEF score was kept and used in a sensitivity analysis.

\section{Assessment of depression}

The presence of depressive disorder during the year after the MI was assessed with the composite international diagnostic interview (CIDI-Auto), a standardised psychiatric diagnostic interview. ${ }^{18}$ The interview was conducted at about three and 12 months after the MI to get a valid assessment of depression during the whole year. Patients were interviewed in their home. Diagnoses were assessed according to International classification of diseases, 10th revision criteria. At the 12 month CIDI-Auto assessment, the duration of post-MI depression was estimated. We recoded the duration of postMI depressive disorder in three categories: $<3$ months, 3-9 months, and $>9$ months. The severity of post-MI depressive symptoms was assessed during hospitalisation and at about three and six months after the MI with the Beck depression inventory (BDI). ${ }^{19}$ The BDI is a widely used 21 item, self report measure of the presence and severity of symptoms of depression. Respondents are instructed to rate each symptom on a $0-3$ scale, with 0 representing absent and $1-3$ representing increasing levels of severity. We used the highest BDI score during follow up as an indicator of the severity of the post-MI depressive episode, categorised as 0-9, $10-19,20-29$, or $>29$. In addition, we assessed whether the patient had experienced a depressive disorder before the MI. At the 12 month CIDI-Auto, the patient reported whether the emotional problems were discussed with a health care professional, whether treatment was offered, and what the treatment comprised.
Assessment of health status

Health status was assessed at about three and 12 months after the MI and consisted of the following.

\section{Health related QoL}

The RAND 36 item health survey consists of 36 items organised into eight scales assessing aspects of health related QoL. ${ }^{20}{ }^{21}$ We used the following six scales: physical functioning, social functioning, role limitations-physical, role limitations-emotional, pain, and general health. We excluded the scales mental health and vitality because of their operational overlap with depression. Each of the six scales was recoded into standardised scores with a scoring range between 0-100 (100 being optimal functioning). We used the Dutch translation, which has been developed and validated in the IQOLA (international quality of life assessment) project. ${ }^{22}$

\section{Cardiac related complaints}

The health complaints scale (HCS) is a self report measure focused on common health complaints of patients with coronary heart disease. ${ }^{23}$ The HCS consists of 12 somatic and 12 cognitive complaints. For this study, to avoid overlap with symptoms included in the diagnosis of depression, we restricted the assessment to the 12 somatic items. In previous studies, ${ }^{23}{ }^{24}$ internal consistency coefficients of 0.89 and 0.91 and a test-retest reliability coefficient of 0.69 (three months interval length) were found. Also, the HCS proved to be sensitive to change in that patients who underwent rehabilitation reported a reduction of complaints compared with a control group.

We used a patient self report questionnaire, the cardiac complaints questionnaire, to assess the most prevalent cardiac symptoms (palpitations, angina, dyspnoea, and fatigue) based on the New York Heart Association classification (0, no complaints; I, complaints only when running or hurrying; II, complaints when walking normally; III, complaints present at rest).

\section{Disability}

In concordance with the work of Broadhead et al, ${ }^{25}$ patients were asked the following in a disability days questionnaire to indicate within a time frame of the preceding month. How many days were you not able to do your daily activities (for example, your work, housework, studies, leisure activities) because of physical or emotional problems? Apart from the above, on how many days were you able to do your daily activities (for example, your work, house work, studies, leisure activities) for less than half because of physical or emotional problems? Both variables (complete disability and partial disability) were dichotomised as disability for less than one week or at least one week during the preceding month.

\section{Data analysis}

We compared patients with versus patients without post-MI depression on health related QoL, health complaints, and disability at the 12 month follow up. For continuous outcome variables with normal distributions (RAND-36 scales and HCS) we tested by means of $t$ tests whether the groups differed significantly on outcomes; for dichotomous outcome variables (cardiac complaints questionnaire, disability), we used $\chi^{2}$ tests. To further study the effects of post-MI depression in relation to age, sex, cardiac condition, history of depression before MI, and health status at three months after an MI, we conducted a series of linear regression analyses for the continuous outcome variables (RAND-36 scales, HCS) and logistic regression analyses for the dichotomous outcome variables (cardiac complaints questionnaire, 
Table 1 Sociodemographic data, baseline cardiac condition, and health status at three months of patients with and without post-myocardial infarction (MI) depression

\begin{tabular}{|c|c|c|c|c|c|c|}
\hline & $\begin{array}{l}\text { No post-MI } \\
\text { depression } \\
(\mathrm{n}=315)\end{array}$ & $\begin{array}{l}\text { Post-MI } \\
\text { depression } \\
(\mathrm{n}=106)\end{array}$ & $\begin{array}{l}\text { Total sample } \\
(\mathrm{n}=421)\end{array}$ & $t / \chi^{2}$ & df & $p$ Value \\
\hline \multicolumn{7}{|l|}{ Sociodemographic data } \\
\hline Age (years) & $61.86(11.29)$ & $58.31(11.43)$ & $61.0(11.4)$ & 2.79 & 419 & 0.005 \\
\hline Women & $18.1 \%$ & $27.4 \%$ & $20.4 \%$ & 4.19 & 1 & 0.04 \\
\hline \multicolumn{7}{|l|}{ Level of education } \\
\hline Low & $66.3 \%$ & $66.0 \%$ & $66.3 \%$ & & & \\
\hline Medium & $19.4 \%$ & $17.9 \%$ & $19.0 \%$ & & & \\
\hline High & $14.3 \%$ & $16.0 \%$ & $14.7 \%$ & 0.25 & 2 & 0.88 \\
\hline Living alone & $13.3 \%$ & $16.0 \%$ & $14.0 \%$ & 0.48 & 1 & 0.48 \\
\hline Smoking & $47.0 \%$ & $61.6 \%$ & $50.8 \%$ & 6.28 & 1 & 0.01 \\
\hline Pre-Ml depression & $6.0 \%$ & $43.4 \%$ & $15.4 \%$ & 84.8 & 1 & $<0.001$ \\
\hline \multicolumn{7}{|l|}{ Cardiac condition } \\
\hline History of MI & $14.0 \%$ & $15.1 \%$ & $14.3 \%$ & 0.08 & 1 & 0.77 \\
\hline Killip class $\geqslant 2$ & $14.0 \%$ & $15.1 \%$ & $14.3 \%$ & 0.08 & 1 & 0.78 \\
\hline LVEF $\leqslant 40 \%$ & $23.9 \%$ & $22.6 \%$ & $23.6 \%$ & 0.07 & 1 & 0.79 \\
\hline Diabetes mellitus & $9.5 \%$ & $9.4 \%$ & $9.5 \%$ & 0.001 & 1 & 0.98 \\
\hline \multicolumn{7}{|c|}{ Health status at three months (health related QoL score) } \\
\hline Physical functioning & $74.6(23.8)$ & $67.4(24.9)$ & $72.8(24.3)$ & 2.61 & 407 & 0.009 \\
\hline Social functioning & $83.8(20.5)$ & $62.6(22.5)$ & $78.4(22.9)$ & 8.87 & 412 & $<0.001$ \\
\hline Role limitations-physical & $64.7(41.3)$ & $25.2(35.8)$ & $54.8(43.5)$ & 8.66 & 407 & $<0.001$ \\
\hline Role limitations-emotional & $79.7(35.8)$ & $44.0(42.8)$ & $70.7(40.7)$ & 8.32 & 408 & $<0.001$ \\
\hline General health & $65.9(20.3)$ & $51.2(18.7)$ & $62.3(20.9)$ & 6.38 & 405 & $<0.001$ \\
\hline Pain & $86.7(18.3)$ & $76.8(20.3)$ & $84.2(19.3)$ & 4.61 & 411 & $<0.001$ \\
\hline \multicolumn{7}{|l|}{ Cardiac related complaints } \\
\hline Somatic complaints & $18.9(6.8)$ & $25.7(8.3)$ & $20.6(7.8)$ & -8.39 & 411 & $<0.001$ \\
\hline Palpitations & $35.1 \%$ & $59.5 \%$ & $41.3 \%$ & 14.49 & 1 & 0.002 \\
\hline Angina & $37.1 \%$ & $57.0 \%$ & $42.3 \%$ & 9.39 & 1 & $<0.001$ \\
\hline Dyspnoea & $45.9 \%$ & $50.6 \%$ & $47.1 \%$ & 0.53 & 1 & $<0.001$ \\
\hline Fatigue & $28.6 \%$ & $55.1 \%$ & $35.3 \%$ & 18.01 & 1 & $<0.001$ \\
\hline \multicolumn{7}{|l|}{ Disability } \\
\hline Complete disability & $13.2 \%$ & $35.4 \%$ & $18.6 \%$ & 23.66 & 1 & $<0.001$ \\
\hline Partial disability & $12.6 \%$ & $42.3 \%$ & $19.9 \%$ & 40.24 & 1 & $<0.001$ \\
\hline
\end{tabular}

disability). Effect sizes were based on the corresponding adjusted unstandardised regression weights (and their 95\% confidence intervals (CIs)) in the linear regression models and on the adjusted odds ratios (ORs) (and their 95\% CIs) in the logistic regression models. In all analyses, we entered post-MI depression, sex, age, history of MI, Killip class, LVEF, pre-MI depression, and corresponding health status indicators at three months after an MI as forced predictors, without interaction terms. The regression weights associated with depression can therefore be interpreted as the strength of the independent effect of depression on changes in health status between 3-12 months while controlling for confounders.

Two sensitivity analyses were conducted to assess the stability of our findings. Firstly, to minimise the potential effects of self report bias due to assessment of depression and health status in the same period of time, we excluded patients with prevalent depression in the 9-12 months after MI and evaluated whether the association between post-MI depression and future health status was still present. Secondly, we minimised the possibility of suboptimal control of a baseline cardiac condition by repeating the multivariate analyses for those patients for whom LVEF was available as a continuous measure.

To study whether characteristics and treatment status of the post-MI depression further contributed to poor health status, we conducted linear and logistic regression analyses of the subgroup of patients with post-MI depression, with duration of the depressive episode, severity of depressive symptoms, and treatment status as independent variables while controlling for age, sex, history of MI, Killip class, LVEF, and pre-MI depression. For all analyses, $\mathrm{p}<0.05$ was considered to indicate significance.

\section{RESULTS}

\section{Patient sample characteristics}

A total of 1166 patients with a confirmed MI fulfilling the inclusion criteria were admitted at the participating wards. Of this sample, 284 patients fulfilled the exclusion criteria: 13 ( $1 \%)$ had a life expectancy less than one year for noncardiovascular reasons, 57 (5\%) had poor cognitive functions, $14(1 \%)$ were unable to speak or read Dutch, 7 (1\%) had visual or auditory problems that precluded participation, 60 (5\%) had an MI during hospital admission for other reasons, $44(4 \%)$ were scheduled for follow up visits in a nonparticipating hospital, $60(5 \%)$ had a too poor physical condition according to the hospital staff, and 29 patients (3\%) died before they could be approached or decide about participation. This resulted in 882 patients whom we asked to participate in the study. We obtained informed consent from 528 patients, 468 of whom completed the psychiatric assessment. Follow up was available for 421 (90.0\%) of the included patients.

On the basis of the information from the CIDI-Auto assessments, 106 respondents $(25.2 \%)$ were found to have a post-MI depressive disorder during the post-MI year and 315 respondents did not. According to their BDI scores, 77 patients $(72.6 \%)$ had depression classified as mild, 20 patients $(18.9 \%)$ as moderate, and nine patients $(8.5 \%)$ as severe. For 39 patients $(36.8 \%)$ the episode lasted less than three months, for $49(46.2 \%)$ it lasted 3-9 months, and for $18(17.0 \%)$ it lasted for more than nine months. A total of 78 patients $(73.6 \%)$ reported having discussed their problems with a health care professional and $32(30.2 \%)$ reported having received some form of treatment, of whom $14(13.2 \%)$ received antidepressant medication. 
Table 2 Health status at 12 months after $\mathrm{Ml}$ in patients with and without post-MI depression

\begin{tabular}{llllll}
\hline & $\begin{array}{l}\text { No post-MI } \\
\text { depression }(\mathbf{n = 3 1 5 )}\end{array}$ & $\begin{array}{l}\text { Post-MI depression } \\
(\mathbf{n = 1 0 6})\end{array}$ & $\mathbf{t} / \chi^{2}$ & df & p Value \\
\hline $\begin{array}{l}\text { Health related QoL } \\
\text { Physical functioning }\end{array}$ & $73.53(24.77)$ & $61.7(26.62)$ & 4.06 & 398 & $<0.001$ \\
Social functioning & $86.43(19.46)$ & $65.12(24.06)$ & 9.13 & 417 & $<0.001$ \\
Role limitations-physical & $72.91(38.98)$ & $37.38(42.34)$ & 7.90 & 414 & $<0.001$ \\
Role limitations-emotional & $83.60(32.91)$ & $48.57(44.58)$ & 8.58 & 416 & $<0.001$ \\
General health & $66.02(19.91)$ & $50.10(19.51)$ & 6.89 & 400 & $<0.001$ \\
Pain & $88.54(18.21)$ & $76.99(21.27)$ & 5.40 & 417 & $<0.001$ \\
Cardiac related complaints & $17.56(5.98)$ & $25.19(8.35)$ & -9.86 & 391 & $<0.001$ \\
Somatic complaints & $30.6 \%$ & $54.7 \%$ & 19.89 & 1 & $<0.001$ \\
Palpitations & $31.1 \%$ & $55.7 \%$ & 20.44 & 1 & $<0.001$ \\
Angina & $42.9 \%$ & $63.2 \%$ & 13.16 & 1 & $<0.001$ \\
Dyspnoea & $25.1 \%$ & $54.7 \%$ & 31.74 & 1 & $<0.001$ \\
Fatigue & $10.6 \%$ & $30.8 \%$ & 23.71 & 1 & $<0.001$ \\
Disability & $12.0 \%$ & $35.3 \%$ & 28.27 & 1 & $<0.001$ \\
Complete disability & & & & &
\end{tabular}

Of the 106 respondents with a post-MI depressive disorder, a considerable proportion reported an additional lifetime anxiety disorder: 42 (39.6\%) had no co-morbid anxiety disorder, $18(17.0 \%)$ had one co-morbid anxiety disorder, and $46(43.4 \%)$ had two or more anxiety disorders. The prevalences of the specific anxiety disorders (without hierarchical exclusion rules applied) were as follows: generalised anxiety disorder, 40 (37.7\%); social phobia, 15 (14.2\%); agoraphobia (with or without panic), 29 (27.4\%); specific phobia, 21 (19.8\%); panic disorder, 14 (13.2\%); and other anxiety disorder, 15 (14.2\%). In addition, 15 (14.2\%) of the depressed patients had dysthymia.

A comparison between these groups on baseline data (table 1) showed significant differences in age, sex, smoking, depression before the MI, and health status at three months. No differences were observed regarding history of MI, Killip class, LVEF, level of education, and living situation.

\section{Effects of post-MI depression on health status at 12 months after MI}

Patients with post-MI depression had a significantly poorer health status on all indicators at 12 months after the MI than patients without a post-MI depression in terms of poorer health related QoL, more self reported cardiac related complaints, and more disability (table 2).

After controlling for age, sex, cardiac condition, and pre-MI depression, all associations with health status at 12 months follow up remained significant. Higher age, female sex, and history of MI were associated with some aspects of poorer health status, whereas the indicators of cardiac condition and being depressed before the MI in general were only marginally related. The magnitude of the adjusted effects of post-MI depression on health status was almost identical to the (unadjusted) differences between depressed and nondepressed patients in table 2, suggesting largely independent effects: physical functioning, -12.6 (95\% CI -18.6 to -6.6$)$; social functioning, $-19.8(95 \%$ CI -24.9 to -14.7$)$; role limitations-physical, -32.1 (95\% CI -41.8 to -22.5 ); role limitations-emotional $-34.0(95 \%$ CI -43.0 to -25.1$)$; general health, -15.4 (95\% CI -20.3 to -10.4$)$; pain, -10.5 (95\% CI -15.2 to -5.9$)$; and HCS-somatic complaints, -6.6 (95\% CI -4.9 to -8.3 ) (all unstandardised corrected $\beta$ weights); palpitations, 2.49 (95\% CI 1.49 to 4.14 ); angina, 2.28 (95\% CI 1.37 to 3.78); dyspnoea, 2.27 (95\% CI 1.35 to 3.81); fatigue, 2.87 ( $95 \%$ CI 1.71 to 4.83 ); complete disability, 4.71 ( $95 \%$ CI 2.47 to 8.98 ); and partial disability, 4.46 (95\% CI 2.40 to 8.27 ) (all multivariate ORs).
Adding the corresponding health status assessed at three months' follow up to the prediction models resulted in significant effects of post-MI depression in all indicators except for palpitations and angina, for which a nonsignificant trend was observed (table 3 ). The magnitude of the effects amounted to about half of those found in the analyses without controlling for health status at three months with respect to QoL and about one third with respect to somatic complaints. No reduction of the effects was found with respect to dyspnoea, fatigue, and disability.

\section{Sensitivity analyses}

Of the 106 patients with a post-MI depression, 36 (34\%) still were depressed in the 9-12 months after their MI. We repeated the multivariate analyses after excluding these patients, resulting in the following estimates of the effects of post-MI depression: physical functioning, -6.97 (95\% CI -12.00 to -1.94$)$; social functioning, -6.55 (95\% CI -11.96 to -1.15$)$, role limitations-physical, -14.73 (95\% CI -25.33 to -4.13 ); role limitations-emotional, -9.25 (95\% CI -18.81 to 0.32 ); general health, -2.79 (95\% CI -7.70 to 2.20$)$; pain, -1.83 (95\% CI -6.53 to 2.81 ); and HCS-somatic complaints, 1.88 (95\% CI 0.44 to 3.33 ) (all unstandardised corrected $\beta$ weights); palpitations, 1.65 (95\% CI 0.72 to 3.79 ); angina, 1.02 (95\% CI 0.44 to 2.32 ); dyspnoea, 1.58 (95\% CI 0.71 to 3.50); fatigue, 1.83 (95\% CI 0.84 to 3.99); complete disability, 4.59 (95\% CI 2.00 to 10.53 ); and partial disability, 2.94 (95\% CI 1.32 to 6.54) (all multivariate ORs). Significant effects of post-MI depression thus remained with respect to physical and social functioning, pain, and disability (complete and part).

Adding LVEF as a continuous score to the regression model instead of the dichotomised score resulted in significant effects of LVEF on social functioning $(p=0.004)$ and partial disability $(p=0.02)$ and the following estimates of the effects of post-MI depression on health status: physical functioning, $-9.02(95 \%$ CI -14.67 to -3.37$)$; social functioning, -9.60 (95\% CI -15.84 to -3.37$)$; role limitations-physical, -9.57 (95\% CI -21.05 to -1.91$)$; role limitations-emotional, -16.47 (95\% CI -27.90 to -5.04$)$; general health, -8.30 (95\% CI -13.28 to -3.31$)$; pain, -6.95 (95\% CI -11.94 to -1.95$)$; and HCS-somatic complaints, 2.97 (95\% CI 1.37 to 4.58 ) (all unstandardised corrected $\beta$ weights); palpitations, 2.08 (95\% CI 0.73 to 5.93); angina, 0.88 (95\% CI 0.33 to 2.34); dyspnoea, 2.10 (95\% CI 0.80 to 5.53); fatigue, 2.14 (95\% CI 0.82 to 5.59 ); complete disability, 5.70 (95\% CI 2.35 to 13.82 ); partial disability, 3.17 (95\% CI 


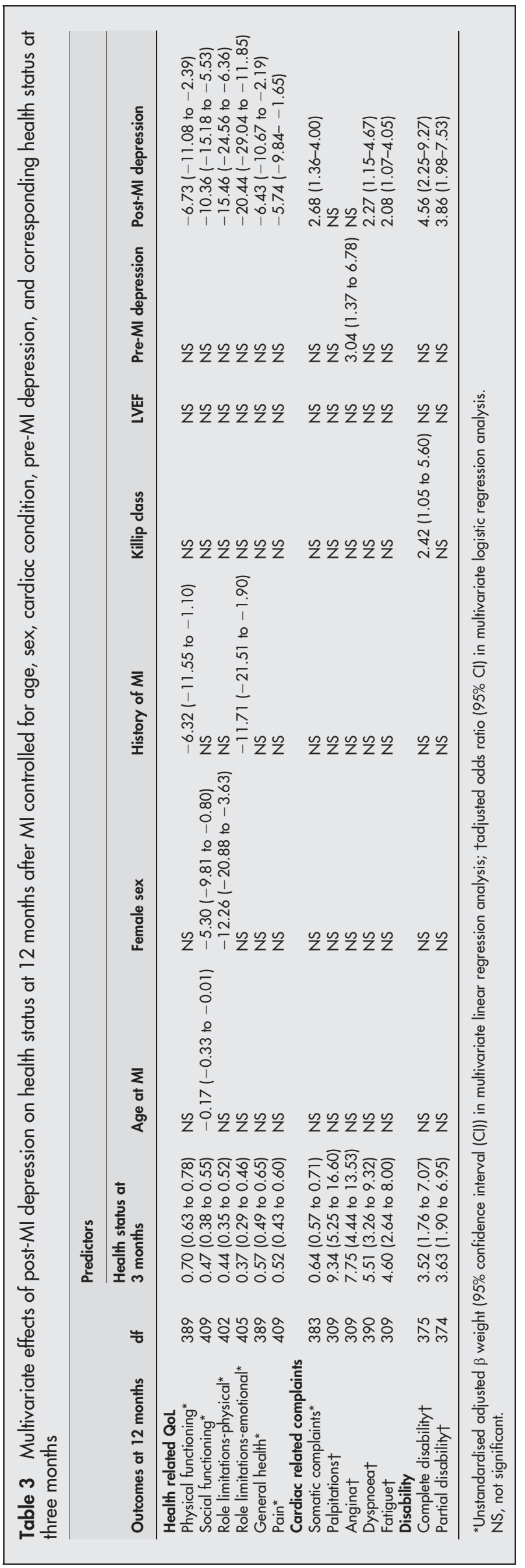

1.35 to 7.47) (all multivariate ORs). Thus, all effects of postMI depression on QoL, somatic complaints and disability remained significant, whereas the effects on cardiac symptoms were not.

The addition of smoking, living situation, and level of education as potential confounders did not alter the significance and magnitude of the effects of depression.

\section{Effects of depression characteristics on health status}

In table 4, severity and duration of the post-MI depression where added to the predictive model of health status at follow up. These analyses were restricted to the subgroup of patients with post-MI depression. Severity of depressive symptoms contributed to a further reduction in QoL, increased reporting of palpitations, and more partial disability. Duration of depression was not associated with outcomes at 12 months except for angina. Treatment status of depression was not significantly associated with any of the health status indices, although favourable trends were observed with respect to social functioning $(4.90,95 \%$ CI -4.67 to 14.49$)$, role-physical functioning $(6.75,95 \% \mathrm{CI}$ -10.47 to 23.97), role-emotional functioning (9.36, 95\% CI -9.56 to 28.29$)$, somatic complaints $(-2.04,95 \%$ CI -5.21 to 1.12 ), angina (OR $0.38,95 \%$ CI 0.09 to 1.63 ), dyspnoea (OR $0.64,95 \%$ CI 0.18 to 2.33 ), and fatigue (OR $0.52,95 \%$ CI 0.15 to 1.82 ).

\section{DISCUSSION}

We assessed the prospective association of post-MI depression with health status in a large sample of patients after an MI. In accordance with previous reports, ${ }^{14}$ we found that post-MI depression is strongly related to poor future health status and that this association is independent of cardiac condition, which was only marginally related. Since we used a prospective design that focused on depressive disorder as assessed with a formal psychiatric interview after hospitalisation and controlled for baseline cardiac condition, history of depression, and health status at three months and conducted sensitivity analyses with respect to the assessment of LVEF and post-MI depression, we conclude that this effect is specific for post-MI depression.

Elsewhere, we have reported the effects of depression before a somatic event, including MI and congestive heart failure, on adjustment after the event. Patients with preevent depressive symptoms were more likely to have a problematic course of functioning and QoL during the year after the event. ${ }^{11}$ In the present study, we found that post-MI depression had a far more profound effect on health status than pre-MI depression. In fact, in univariate analyses we found that pre-MI depression was associated with poor health status at follow up, but in multivariate analyses this association was overshadowed by the far stronger association of post-MI depression with health status and the association between pre-event and post-event depression. The observation that a substantial proportion $(43.4 \%)$ of the post-MI depressed patients had experienced a pre-MI depressive episode, however, suggests an intriguing longitudinal bidirectional association between heart disease and depression, with poor health status as outcome. The effects of post-MI depression on health status at 12 months were reduced for most of the indicators when health status at three months was added to the prediction models but remained present especially with respect to QoL and disability. This finding indicates that depression not only is associated with poor health status but also predicts changes in (aspects of) health status. On the other hand, our data suggest that previous reports that did not correct for baseline health status may have overestimated the effects of depression. ${ }^{14}{ }^{15}$ Similarly, for several of the health status indicators we found that the 


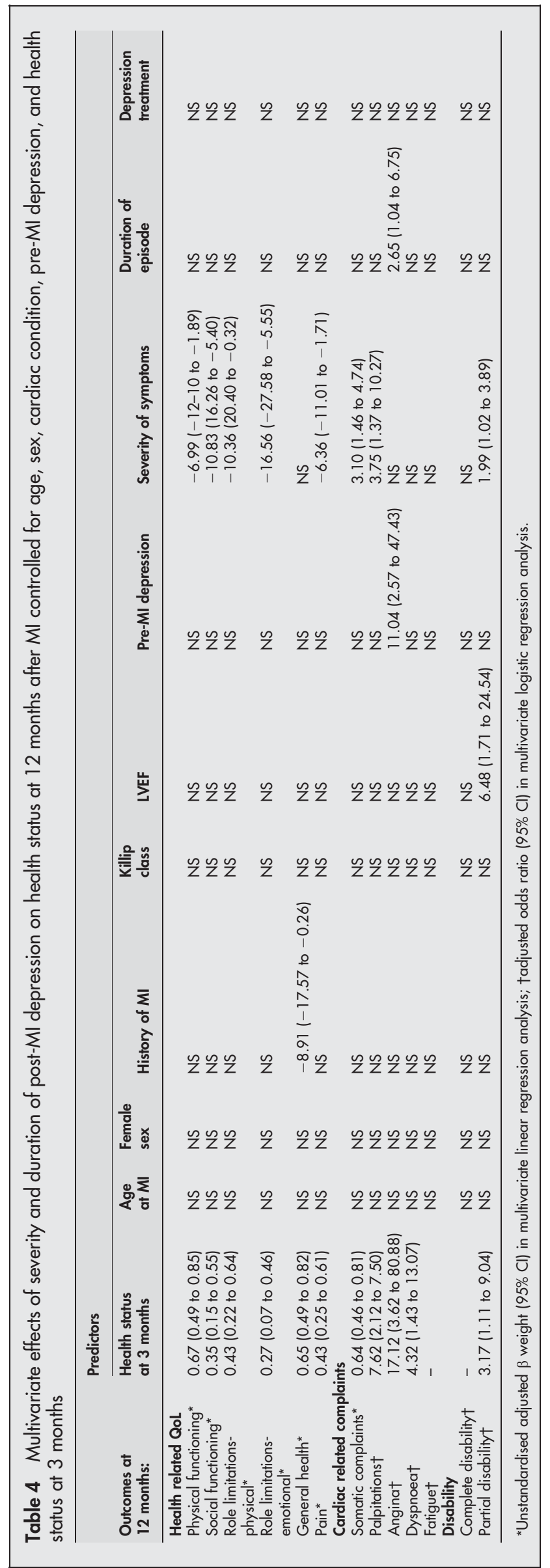

effects of post-MI depression were attenuated by depressive symptoms still present at the follow up assessment. In other words, some authors may well have overestimated the prospective effects of depression on health status because respondents had a concurrent depression that affected health status or its assessment. As an illustration, in our sample we found that one third (36 of 106) of post-MI depressed patients were (still) depressed at 9-12 months after an MI, due to a long duration of depression (18 patients) or a relatively late onset of post-MI depression (another 18 patients).

Among the limitations of the study, firstly, the considerable proportion of excluded patients during the phases of the study should be considered. The number of patients who did not give informed consent, had incomplete assessments, or were lost to follow up may have resulted in an under representation of patients with poor health status. To see whether our study was still representative of the population, we compared our data with results reported from previous studies. Generally, an estimated prevalence of $15-25 \%$ of post-MI depression is reported, so our finding of $25.2 \%$ ( 106 of 421 ) seems in line. In the existing literature, about $5 \%$ of patients died of cardiac related causes during follow up (mean $1-1.5$ years). In our sample this was eight of 421 after 2.5 years $(2 \%)$, which is somewhat lower. Our sample thus apparently has a more favourable prognosis than previous samples, which may be due to the participation rate or to improved care; it is not clear what influence this has had on our results. We would expect the group of patients who refused to participate to consist of patients with poor cardiac function and to be at high risk of poor health status. Excluding them would then result in an underestimation of the effect of cardiac functioning and depression on health status but not so much on the independent effect of depression on health status, which was the primary goal of the study. Secondly, since we did not have continuous LVEF scores for all patients, the adjustment for baseline cardiac condition may not have been optimal. However, controlling for LVEF as a continuous score for the patients for whom this was available $(63.4 \%)$ did not alter the magnitude of the effects of depression on health status. As a third potential study limitation, we note that our health status assessments depend on self report data, which provided us with a less standardised measure of functioning than, for example, treadmill performance tests. This way a bias may have been introduced with depression colouring the patients' self reports. We limited this potential bias by adding specific measures of specific complaints (for example, palpitations) and real life events (for example, disability days) to the generic health status indices (for example, RAND-36) by assessing depression with a formal psychiatric interview instead of a self report questionnaire and, most important, by correcting for self reported health status at three months. This correction led to a reduction of the estimated effects of depression on health status but it remained present for most of the indicators. Furthermore, we performed secondary analyses excluding patients whose depression persisted until the health status assessment at 12 months after MI. We found that post-MI depression remained associated with health status at 12 months even after excluding these patients, although the magnitude of the effects was reduced somewhat.

Depression is expected to have an enormous impact on the worldwide burden of disease in the coming years. ${ }^{26}$ The diseases with the strongest impact are predicted to be ischaemic heart diseases and major depression because of their high prevalence (about 5\% of the population is depressed) and their strong effects on QoL. However, unlike patients with ischaemic heart diseases, about half of the 
people with a somatic illness who are depressed receive no or inadequate treatment, ${ }^{27}{ }^{28}$ despite the availability of interventions with proven efficacy. ${ }^{29}{ }^{30}$ Given the high prevalence of post-MI depression-about 15-25\% of patients develop major depression in the year after an MI and a similar proportion develop minor depression ${ }^{5-7}$ - the question becomes pertinent whether screening for depression and its subsequent treatment should become standard practice in the aftercare of MI. Of the post-MI depressed patients, only a minority were treated. The present study does show the potential gain of effective treatment of depression after MI on health status: post-MI depression was associated with a twofold increased risk of dyspnoea and fatigue, a fourfold increased risk of disability, and a $0.3-0.5$ standard deviation difference on the QoL scales, corresponding to a moderate effect. ${ }^{31}$

Of interest, in this study we assessed depression by means of a standardised psychiatric interview with patients after discharge from the hospital, which results in an estimation of the presence of a treatable disorder, rather than self reported symptoms that may be confounded by symptoms related to the MI. Also, out study shows the potential use of the BDI as an easy to administer self report screening instrument to determine the level of depressive complaints, since we found that even within the subgroup of depressed patients, the BDI score was associated with poorer health status. Still, the extent to which instruments to assess depression are applicable to depression after MI is debated. In a recent study, we found that post-MI depression consisted of two correlated symptom factors. Specifically, the dimension of self reported cognitive/affective symptoms was not related to somatic health and cardiovascular prognosis, whereas the dimension of somatic/affective symptoms was confounded by somatic health status and yet independently associated with cardiovascular prognosis. ${ }^{32}$

Recently, sertraline was shown to be more efficacious than placebo in reducing depressive symptoms after $\mathrm{MI}^{33}{ }^{34}$ and cognitive behavioural therapy as standard treatment for postMI depression was shown to be associated with reduced depression when compared with care as usual. ${ }^{35}$ It is still not clear whether effective treatment of depression also improves cardiac outcomes and health status. The present study was not designed to assess the effects of antidepressant treatment on health status but we did observe some favourable nonsignificant trends in that patients receiving some form of antidepressant treatment tended to report fewer complaints and higher QoL. To examine the effectiveness of antidepressant treatment of post-MI depression, this question has to be addressed in a randomised controlled trial. ${ }^{36}$

\section{ACKNOWLEDGEMENTS}

The DepreMI study was funded by a grant from the Netherlands Organisation for Scientific Research.

Participating hospitals, cardiologists, and researchers: Academisch Ziekenhuis Groningen: T A Spijkerman, R H S van den Brink, J F May, J B Winter, J H C Jansen, H J G M Crijns, and J Ormel. Martini Ziekenhuis Groningen: J H Bennekers, F van den Berg, P J L M Bernink, R B van Dijk, M G Niemeyer, J L Postma, L E J M Schrijvers, and L H Takens. Refaja Ziekenhuis Stadskanaal: K de Vries and L M van Wijk. St Lucas Ziekenhuis Winschoten: T R Bouwmeester and A van der Galien.

\section{Authors' affiliations \\ P de Jonge*, T A Spiikermant, R H S van den Brinkt, J Ormelt, Department of Psychiatry, University of Groningen, Groningen, the Netherlands \\ *Also Graduate Research School Behaviour, Cognition and Neurosciences (BCN), Graduate Research School Experimental Psychopathology (EPP), and the Department of Internal Medicine, Academic Hospital Groningen \\ †Also BCN and EPP}

\section{REFERENCES}

1 Ruo B, Rumsfeld JS, Hlatky MA, et al. Depressive symptoms and health-related quality of life: the heart and soul study. JAMA 2003;290:215-21.

2 Rumsfeld JS. Health status and clinical practice: when will they meet? Circulation 2002;106:5-7.

3 Gorkin L, Follick MJ, Geltman E, et al. Quality of life among patients postmyocardial infarction at baseline in the survival and ventricular enlargement (SAVE) trial. Qual Life Res 1994;3:111-9.

4 Mattera JA, De Leon CM, Wackers FJ, et al. Associations of patients' perception of health status and exercise electrocardiogram, myocardia perfusion imaging, and ventricular function measures. Am Heart $J$ 2000;140:409-18.

5 Lane D, Carroll D, Lip GYH. Psychology in coronary care. Q J Med 1999:92:425-31.

6 Frasure-Smith N, Lesperance F, Talajic M. Depression following myocardial infarction: impact on 6-month survival. JAMA 1993;270:1819-25.

7 Schleifer SJ, Macari HM, Coyle DA, et al. The nature and course of depression following myocardial infarction. Arch Intern Med 1989;149:1785-9.

8 Forrester AW, Lipsey JR, Teitelbaum ML, et al. Depression following myocardial infarction. Int J Psychiatry Med 1992;22:33-46.

9 Ormel J, Kempen GIJM, Deeg D, et al. Functioning, well-being, and health perception in late middle-age and older people: comparing the effects of depressive symptoms and chronic medical conditions. J Am Geriatr Soc 1998;46:39-48.

10 Wells KB, Stewart A, Hays RD, et al. The functioning and well-being of depressed patients: results from the medical outcomes study. JAMA 1989;262:914-9.

11 De Jonge P, Ormel J, Slaets JPJ, et al. Depressive symptoms in the elderly predict poor adjustment following somatic events. Am J Geriatr Psychiatry 2004; 12:57-64.

12 Spertus JA, McDonell M, Woodman CL, et al. Association between depression and worse disease-specific functional status in outpatients with coronary artery disease. Am J Cardiol 2000;140:105-10.

13 Sullivan MD, LaCroix AZ, Baum C, et al. Functional status in coronary artery disease: a one-year prospective study on the role of anxiety and depression. Am J Med 1997; 103:348-56.

14 Lane D, Carroll D, Ring C, et al. Mortality and quality of life 12 months after myocardial infarction: effects of depression and anxiety. Psychosom Med $2001 ; 63: 221-30$

15 Lane D, Carroll D, Ring C, et al. Effects of depression and anxiety on mortality and quality-of-life 4 months after myocardial infarction. J Psychosom Res 2000:49:229-38

16 Macleod J, Davey Smith G, Heslop P, et al. Limitations of adjustment for reporting tendency in observational studies of stress and self reported coronary heart disease. J Epidemiol Community Health 2002;56:76-7.

17 Watson D, Pennebaker JW. Health complaints, stress and distress: exploring the role of negative affectivity. Psychol Rev 1989;96:234-54.

18 Wittchen HU. Reliability and validity studies of the WHO-composite international diagnostic interview (CIDI): a critical review. J Psychiatr Res 1994;28:57-84.

19 Beck AT, Steer RA. Beck depression inventory manual. San Antonio: Harcourt Brace Jovanovich, 1987

20 Ware JE, Snow KK, Kosinski M, et al. SF-36 health survey manual and interpretation guide. Boston: New England Medical Center, 1993.

21 Keller SD, Bayliss MS, Ware JE, et al. Comparison of responses to SF-36 health survey questions with one-week and four-week recall periods. Health Serv Res 1997:32:367-84

22 Essink-Bot ML, Krabbe PFM, Bonsel GJ, et al. An empirical comparison of four generic health status measures. Med Care 1997;35:522-37.

23 Denollet J. Health complaints and outcome assessment in coronary heart disease. Psychosom Med 1994;56:463-74.

24 Pedersen SS, Denollet J. Perceived health following myocardial infarction: cross-validation of the health complaints scale in Danish patients. Behav Res Ther 2002;40:1221-30.

25 Broadhead WE, Blazer DG, George LK, et al. Depression, disability days, and days lost from work in a prospective epidemiologic survey. JAMA 1990;264:2524-8.

26 Murray C, Lopez A. Alternative projections of mortality and disability by cause 1990-2020: Global burden of disease study. Lancet 1997;349:1498-504.

27 Kessler D, Lloyd K, Lewis $G$, et al. Cross sectional study of symptom attribution and recognition of depression and anxiety in primary care. BMJ 1999:318:436-9

28 Penn JV, Boland R, McCartney JR, et al. Recognition and treatment of depressive disorders by internal medicine attendings and housestaff. Gen Hosp Psychiatry 1997; 19:179-84.

29 Gill D, Hatcher S. A systematic review of the treatment of depression with antidepressant drugs in patients who also have a physical illness, Cochrane Depression and Neurosis Collaborative Review Group. Oxford: Institute of Health Sciences, 1998.

30 Voellinger R, Berney A, Baumann P, et al. Major depressive disorder in the general hospital: adaptation of clinical practice guidelines. Gen Hosp Psychiatry 2003;25:185-93.

31 Cohen J. Statistical power analysis for the behavioral sciences, 2nd ed. Hillsdale: Lawrence Earlbaum Associates, 1988.

32 De Jonge $\mathbf{P}$, Ormel J, Van den Brink RH, et al. Dimensions of depression following myocardial infarction and their relationship with somatic health status and cardiovascular prognosis. Am J Psychiatry (In press). 
33 Glassman $\mathrm{AH}, \mathrm{O}^{\prime}$ Connor CM, Califf RM, et al. Sertraline treatment of major depression in patients with acute $\mathrm{Ml}$ or unstable angina. JAMA 2002;288:701-9.

34 Swenson JR, O'Connor CM, Barton D, et al. Influence of depression and effect of treatment with sertraline on quality of life after hospitalization for acute coronary syndrome. Sertraline Antidepressant Heart Attack Randomized Trial (SADHART) Group. Am J Cardiol 2003;92:1271-6.
35 Berkman LF, Blumenthal J, Burg $M$, et al. Effects of treating depression and low perceived social support on clinical events after myocardial infarction: the enhancing recovery in coronary heart disease patients (ENRICHD) randomized trial. JAMA 2003;289:3106-16.

36 Van den Brink RH, Van Melle JP. Honig A, et al. Treatment of depression after myocardial infarction and the effects on cardiac prognosis and quality of life: rationale and outline of the myocardial infarction and depression-intervention trial (MIND-IT). Am Heart J 2002;144:219-25.

\section{IMAGES IN CARDIOLOGY}

\section{Coronary air embolism after removal of central venous catheter}

doi: $10.1136 /$ hrt.2005.066084

A 39 year old woman with bronchial asthma collapsed following an acute asthma attack. She was admitted to the medical intensive care unit in status asthmaticus requiring mechanical ventilation and severe anoxic encephalopathy. On the 10th hospital day a right subclavian catheter was removed. Within three minutes severe bradycardia was noticed associated with pronounced ST elevation in the inferior wall (panel A) and prominent ST depression in Ll and avL (precordial leads are not available). The patient was placed on 100\% oxygen, and in the Trendelenburg and left lateral decubitus position. ST elevation and bradycardia completely resolved within five minutes (panel B). Cardiac troponin $\mathrm{T}$ and creatine phosphokinase remained normal.

Air embolus during the insertion or removal of central venous catheters and positive pressure mechanical ventilation is an increasingly recognised problem. The exact frequency of this potentially fatal complication is unknown and difficult to assess. It is believed that many unexplained decompensations in critically ill patients may be attributed to air embolisation. Manifestations are predominantly respiratory, cardiovascular, and neurologic. Once suspected, patients should be placed on $100 \%$ oxygen to facilitate gas absorption and in the Trendelenburg left lateral decubitus position to prevent air migration into the pulmonary circulation and systemic embolisation. The present case probably illustrates a rather rare manifestation of air embolus into the coronary circulation resulting in severe transient myocardial ischaemia.

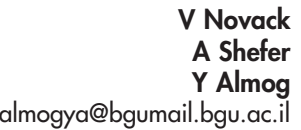

A

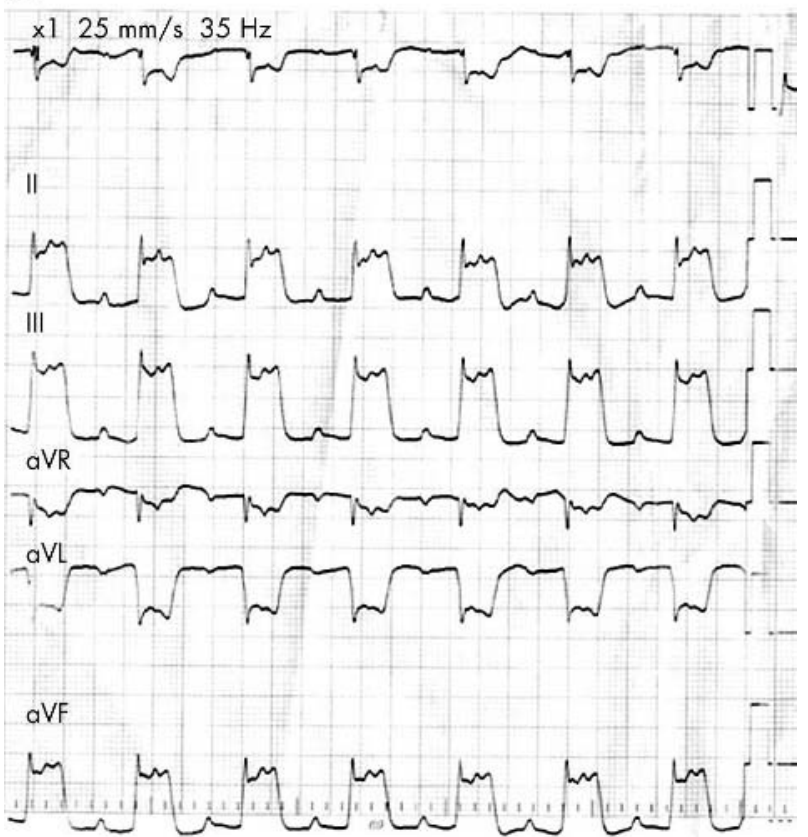

A limb lead ECG, performed within three minutes, showing pronounced $12 \mathrm{~mm}$ ST elevation in the inferior wall and $10 \mathrm{~mm}$ ST depression in L1 and avL.

\section{B}

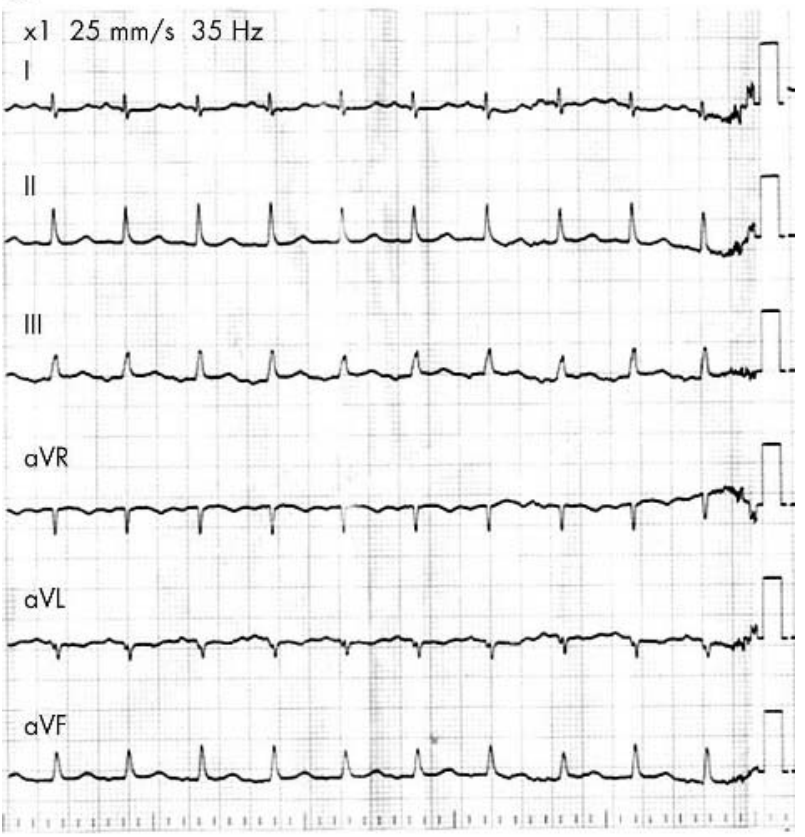

A limb lead ECG, performed 10 minutes later, showing normalisation of ST segments. 